\title{
Sistema para Controle e Monitoramento de Ambientes Utilizando Internet das Coisas
}

\author{
Luciana S. Amorim* Bernardo A. G. Oliveira* \\ Thiago M. Machado-Coelho** Gustavo L. Soares* \\ Zélia M. A. Peixoto* \\ * Programa de Pós-Graduação em Engenharia Elétrica, Pontifícia \\ Universidade Católica de Minas Gerais, Belo Horizonte - MG (e-mail: \\ lucianamorimm@gmail.com; bernardo.godinho@sga.pucminas.br; \\ gsoares@pucminas.br; assiszmp@pucminas.br) \\ ** Programa de Pós-Graduação em Engenharia Elétrica, Univerisdade \\ Federal de Minas Gerais, Belo Horizonte -MG (e-mail: \\ thmmcoelho@ufmg.br)
}

\begin{abstract}
The increase in demand for convenience and security, together with the focus on energy efficiency, has made residential automation become more relevant. One way to expand the use this technology is by applying the Internet of Things (IoT) concept, that connects things and people through wireless networks. The objective of this work is to develop a system that monitors and controls residential environments by using the IoT concept. The developed devices are independent and used in a cloud system in order to perform their tasks, which can be done remotely. Services that allow integration with external systems are available through the rest API.

Resumo: Com o aumento da demanda por comodidade, segurança e o foco em eficiência energética, a automação residencial vem ganhado espaço. Uma aposta para expandir o uso desta tecnologia e melhorar a questão energética é a aplicação do conceito de Internet das Coisas (IoT), que interliga coisas e pessoas através de redes sem fio. O objetivo deste trabalho é desenvolver um sistema para controle e monitoramento de ambientes utilizando o conceito de IoT. Os dispositivos construídos são independentes e estão integrados a um sistema nas nuvens para realização do procedimento, que pode ser feito de modo remoto. São disponibilizados serviços que permitem a integração de sistemas externos através de um web server criado com API rest.
\end{abstract}

Keywords: Home Automation; Control of Environments; Internet of Things; Energy Efficiency; Integrated System.

Palavras-chaves: Automação Residencial; Controle de Ambientes; Internet das Coisas; Eficiência Energética; Sistema Integrado.

\section{INTRODUÇÃO}

A tecnologia está presente no dia a dia das pessoas e vem conquistando mais espaço porque ela se desenvolve no sentido de melhorar a qualidade de vida. A ideia de Internet das Coisas (IoT) é conectar seres humanos, coisas, dispositivos e sistemas. O principal objetivo é "fazer com que as coisas ou objetos possam ser conectados através da internet, redes de sensores sem fio e telefones inteligentes para que eles possam compartilhar informações automaticamente" (Jain et al., 2012).

Com o advento de novas tecnologias, surge o problema do aumento do consumo energético, e o setor residencial demanda parcela significativa da energia elétrica produzida (Rocha Filho et al., 2018). Somado a isso, o envelhecimento da população exige residências mais adaptadas à nova realidade. Nesse contexto, a automação residencial, sobretudo

\footnotetext{
^ O presente trabalho foi realizado com apoio da Coordenação de Aperfeiçoamento de Pessoal de Nível Superior - Brasil (CAPES).
}

com foco na eficiência energética, vem sendo estudada e implantada. De modo geral, a automação residencial pode proporcionar conforto e economia de energia (Treter et al., 2014). No entanto, ainda existem muitos desafios para ampliação da instalação dessa tecnologia.

Brush et al. (2011) listaram que as principais barreiras a serem superadas são o custo elevado, a pouca flexibilidade do sistema, o gerenciamento insatisfatório e segurança questionável. A pesquisa realizada sugeriu ainda a necessidade de melhorias quanto à mudança estrutural para instalar a tecnologia e que os mecanismos de segurança deveriam ser mais simples e confiáveis. Buscando solucionar esses problemas e expandir o uso da automação residencial, pesquisas apontaram para o uso da IoT e dos sistemas ciber-físicos (Siano et al., 2013).

Este trabalho tem como objetivo o desenvolvimento de um sistema para controle e monitoramento de ambientes utilizando o conceito de IoT. Serão criados dispositivos para controle de lâmpadas, ventiladores e para medição 
de umidade, temperatura, gás e presença. Estes dispositivos serão independentes e estarão comunicados com um sistema nas nuvens para realização do procedimento, que pode ser controlado de maneira remota. A plataforma desenvolvida deve permitir ainda a integração de sistemas externos através de web server.

O restante do trabalho está organizado da seguinte maneira: a Seção 2 apresenta a revisão bibliográfica e a Seção 3, a metodologia proposta. Na Seção 4, são apresentados os resultados e, por fim, na Seção 5, são apresentadas as conclusões e sugestões de trabalhos futuros.

\section{REVISÃO BIBLIOGRÁFICA}

\subsection{Automação residencial}

A automação residencial surgiu para facilitar e automatizar tarefas do dia-a-dia. Nela, o usuário envia comandos para controlar as funções da casa. Um termo mais abrangente é a chamada domótica inteligente, pois a mesma é baseada no comportamento dos habitantes (Sgarbi e Tonidandel, 2006).

De modo geral, existem dois tipos de arquitetura para domótica: arquitetura baseada em automação, onde o usuário se adapta a automação implantada, e arquitetura baseada em comportamento, onde os sistemas devem se adaptar e aprender com os usuários (Lins e Moura, 2010).

Monteiro et al. (2015) propuseram uma arquitetura para um sistema de controle de domótica que faz uma divisão entre a componente lógica e a componente física do sistema. A ideia é um sistema distribuído, autônomo, flexível e seguro no caso de falhas. Além disso, a possibilidade de automatizar cada funcionalidade da casa por vez (proposta na arquitetura) trás benefícios econômicos ao cliente.

\subsection{Internet das Coisas}

A internet teve seu primeiro grande passo na escala evolutiva com a "Internet dos Computadores", passando pela "Internet das Pessoas" e agora é a vez da "Internet das Coisas" (Jain et al., 2012).

O termo Internet das Coisas, do inglês Internet of Things (IoT), se refere a um padrão que conecta, através de rede sem fio, conjuntos de objetos físicos embarcados capazes de se comunicarem para realizar variadas funções (Coetzee e Eksteen, 2011).

Os principais desafios encontrados para IoT são em áreas de confiança, padronização e governança(Coetzee e Eksteen, 2011). Além disso, existem desafios quanto à interoperabilidade, escalabilidade, provisão de abstração, interação espontânea, infraestrutura unificada, multiplicidade, segurança e privacidade (Chaqfeh e Mohamed, 2012; Lin et al., 2017).

A comunicação necessária entre as coisas ou objetos, realizada entre as interfaces da rede, abre possibilidades de ataques externos e internos ao sistema. Existem dois tipos principais de ataques à IoT (Jain e Kajal, 2015): os ataques ativos e os ataques passivos. Nos ataques ativos, alguma informação prejudicial é injetada na rede, que provoca erros no funcionamento dos outros nós ou operação da rede.
Nos ataques passivos, os nós maliciosos não desempenham corretamente suas tarefas, diminuindo o desempenho da rede. Grande parte dos ataques externos podem ser evitados com o uso de cifras e códigos de autenticação de mensagens (Karlof e Wagner, 2003). No entanto, ainda existe o problema dos ataques disparados por adversários internos, ou seja, por aqueles cujas credenciais de acesso são válidas. "As mensagens trocadas por esses adversários são legítimas na medida em que elas são autenticadas com sucesso pelos seus destinatários" (Chess e West, 2007). Isso, contudo, não as torna menos prejudiciais. A Internet das Coisas requer soluções de segurança feitas sob medida. Assim, ataques de espionagem, personificação e retransmissão podem ser eficientemente evitados (Karlof e Wagner, 2003). A solução proposta por Teixeira et al. (2014), denominada Siot (Secure IoT), pressupõe que as mensagens trocadas na rede sejam autenticadas e os sistemas executados pelos nós sejam conhecidos e estejam íntegros. Assim, o invasor tem acesso como um usuário comum, mas não é capaz de trocar seu código ou injetar mensagens falsas diretamente no canal de rede.

\subsection{Aplicação da Internet das Coisas em casas, cidades e redes inteligentes}

O trabalho de Ferreira e Godoy (2016) proporcionou a integração da IoT á tecnologia Zigbee para obter eficiência energética nos sistema de iluminação e refrigeração predial. O objetivo é monitorar e controlar a operação desses sistemas, verificando o horário que tais dispositivos devem ser ligados de acordo com a presença de pessoas, evitando assim o acionamento desnecessário.

Pensando no crescimento da população idosa e que necessita de cuidados constantes, Nath et al. (2018) desenvolveram um sistema para ser integrado nas casas inteligentes que permite localizar o paciente baseado em sua voz. Com isso, o cuidador tem informações em tempo real sobre o paciente. O sistema foi pensado usando o conceito de IoT.

Rocha Filho et al. (2018) criaram o STORm, solução inteligente para tomada de decisões que utiliza computação em névoa e inteligência computacional. O STORm utiliza um comitê de classificadores para tomar as decisões e a computação em névoa para gerenciar e processar as funções residenciais. Para validação, foram feitas aplicações em iluminação e eletrodomésticos. Os resultados mostraram um aprendizado de máquina preciso e estável. Isso porque a computação em névoa conta com arquitetura distribuída e perto dos usuários finais, fornecendo respostas rápidas e de qualidade (Lin et al., 2017).

Ejaz et al. (2017) buscaram eficiência energética em cidades inteligentes. Para isso, realizaram dois estudos de caso para aplicar o conceito de IoT. O primeiro estudo foi em casas automatizadas e o segundo em cidades inteligentes. O resultado mostrou otimização no uso da energia com utilização da IoT. O trabalho indicou ainda caminhos para implantação do uso da IoT: utilização de protocolos leves, otimização de agendamento e modelos preditivos para consumo de energia e abordagem baseada na nuvem, entre outros.

Sakurai et al. (2016) apresentaram um estudo de caso para aplicação de IoT numa linha produtiva, objetivando 
interação entre peças, máquinas e controladores por meio de sensores de identificação por rádio frequência (Radio Frequency Identification - RFID), que foi aplicado à um sistema de produção modular da Festo. O foco do trabalho é a chamada indústria 4.0 ou quarta revolução industrial, que une conceitos como IoT e Cyber-Physical Systems (sistemas ciber-físicos) para melhorar a gestão e o controle da produção. Bigheti et al. (2016) também trabalharam com a ideia da indústria 4.0 e apresentaram um estudo de caso para automação e controle de processo na nuvem. Foi apresentado um controle via rede de uma malha de vazão de uma planta piloto de instrumentação do SENAI. O controlador obtém os dados da vazão no servidor e é capaz de controlar a planta de maneira remota.

\section{METODOLOGIA}

O desenvolvimento do projeto de controle de ambientes utilizando o conceito de Internet das Coisas segue o diagrama apresentado na Figura 1.

O sistema foi desenvolvido usando a notação de objetos JavaScript (JavaScript Object Notation - JSON) devido a sua capacidade de estruturar informações de forma compacta, tornando mais rápida a análise das informações. Além disso, é composta por estruturas de dados universais e virtualmente todas as linguagens de programação moderna as suportam.

Para resolver a questão da segurança foi utilizada a autenticação com tokens. Um token é usado para autenticar o usuário e as permissões que ele possui. O usuário provê um nome de usuário e uma senha no formulário de login e, assim que a requisição é feita, valida-se o usuário no back-end consultando sua existência na base de dados. Se a requisição for válida, cria-se um token e este é retornado no cabeçalho de resposta para que possa ser guardado no navegador usando armazenamento local. Provê-se um token em cada cabeçalho de requisição para áreas protegidas na aplicação. Há ainda um token criptografado para o equipamento. Uma vez que o token obtido é válido, permite-se o acesso à área restrita requisitada e aos equipamentos, retornando a resposta em formato JSON.

O sistema desenvolvido objetiva ainda a integração de sistemas externos (usando ou não a interface aqui desenvolvida). Para isso, seu desenvolvimento é orientado a serviços, de modo que o front-end se comunica com o back-end através de um web server criado com Application Programming Interface (API) rest. O cliente manda uma requisição HyperText Transfer Protocol (HTTP) e o servidor retorna os dados no formato JSON. Desta maneira, uma vez que o cliente está cadastrado no sistema, ele terá permissão para acessar os serviços através da sua própria interface.

\subsection{Dispositivos}

O trabalho propõe dispositivos para controle de lâmpadas, ventiladores e sensores de umidade, temperatura, gás e presença.

Foi escolhido o sensor DHT11 para umidade e temperatura. Ele permite fazer leituras de temperaturas entre 0 e $50^{\circ} \mathrm{C}$ e leituras de umidade entre 20 e $90 \%$ da unidade relativa (UR). A precisão da medição de temperatura é $\pm 2{ }^{\circ} \mathrm{C}$ e a precisão da medição de umidade é $\pm 5 \%$ da UR. O tempo de resposta é em torno de 2 segundos.

Para medição de gás foi utilizado o sensor MQ-2, capaz de detectar concentrações de gases combustíveis e fumaça no ar. Quando o sensor é exposto a um local com gás GLP, sua resistência aumenta. O envio de sinais para o microcontrolador pode ser feito pela saída digital ou pela saída analógica. A saída digital pode ser usada para acionar uma sirene ou acionar um relé que vai fechar/abrir uma porta ou cortar o fornecimento de gás. Já a saída analógica informa o nível de concentração de gases detectados pelo sensor.

O sensor de presença e movimento escolhido foi o PIR DYP-ME003. Ele é um sensor infravermelho que consegue detectar o movimento de objetos que estejam em um raio de até 7 metros. Caso algo ou alguém se mova neste raio, o pino de alarme é ativado. Quando a saída é acionada pelo movimento detectado, esta ficará ativada por um tempo, mesmo que não haja mais movimento. É possível ajustar a duração do tempo de espera para estabilização do PIR através do potenciômetro. A estabilização pode variar entre 5 e 200 segundos. O tempo de bloqueio é aproximadamente 2,5 segundos.

Foram usados dois tipos de atuadores: relés e LED infravermelho. O relé escolhido foi o modelo SRD-05VDCSL-C (Songle). Esse modelo foi utilizado com o objetivo de acionar circuitos de altas tensões com comandos de baixas tensões realizados pelo controlador. Seu acionamento é feito com contatos normalmente abertos (NA). O LED infravermelho utilizado foi o T-1 3/4, que pode ser aplicado em sistemas de transmissão ao ar livre, unidades de controle remoto infravermelho com exigência de alta potência, detector de fumaça e sistemas com aplicação de infravermelho.

A comunicação dos sensores e atuadores com o servidor foi feita com o auxílio do módulo wireless ESP8266, um System-On-Chip (SOC) de baixo consumo de energia e alto desempenho wireless. Este módulo pode trabalhar como um Acess Point (Ponto de Acesso) ou como uma Station (Estação), enviando e recebendo dados. Neste trabalho, ele proporciona a comunicação dos periféricos com a internet, tornando possível a utilização do protocolo Message Queuing Telemetry Transport (MQTT) pra comunicação em tempo real com o sistema. Foi utilizado o ESP826612 (modelo 12), pois neste modelo é disponibilizada uma porta com conversor $\mathrm{AD}$ que será utilizada para aquisição de dados do sensor de gás, temperatura e umidade. Com o modo universal, obtém-se também os dados do sensor de presença. Para o controle das lâmpadas e ventiladores, o ESP funciona apenas como elemento intermediador entre o comando e o atuador.

\subsection{Back-end}

Back-end é a parte do sistema responsável pelas regras de negócios, webservices e APIs de uma aplicação. Corresponde ao lado do servidor.

O elemento central de mediação do back-end é o Node.js, uma plataforma para desenvolvimento de aplicações construída sobre o motor JavaScript do Google Chrome para 
BACK-END

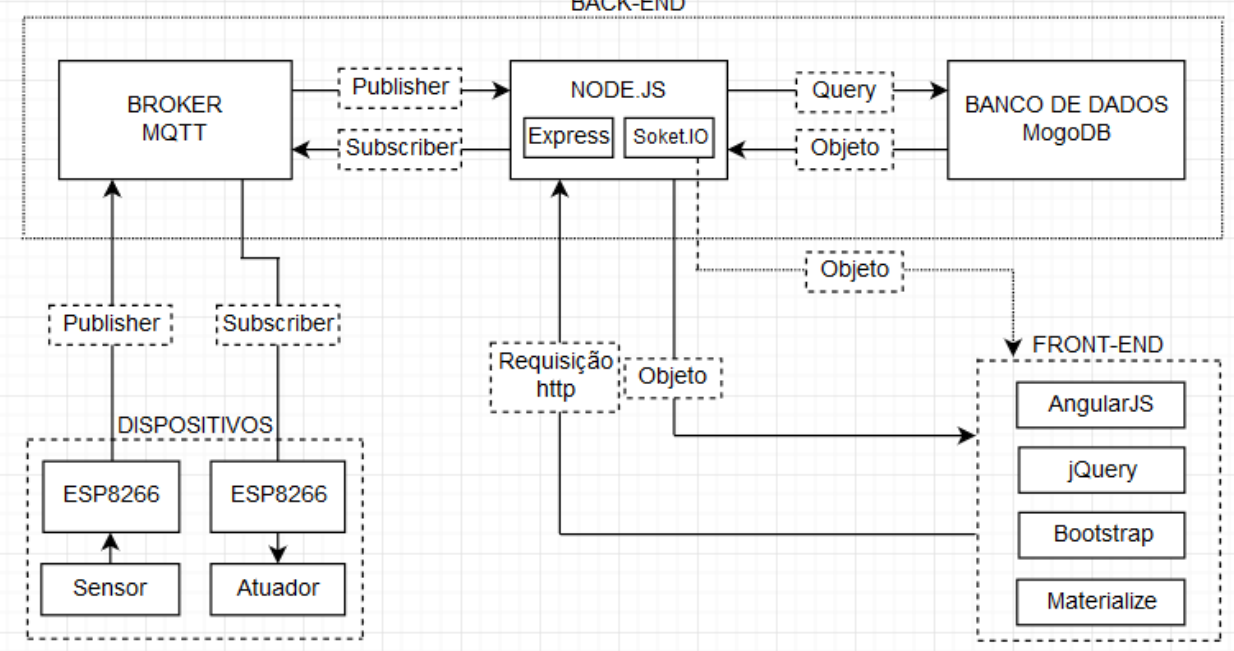

Figura 1. Diagrama de blocos do projeto.

construir aplicações de rede rápidas e escaláveis. É implementado com foco no desempenho e na baixa memória de consumo. Usa o modelo de Input/Output (I/O) direcionada a evento não bloqueante, o que o torna leve e ideal para aplicações em tempo real com troca intensa de dados através de dispositivos distribuídos. Chaniotis et al. (2015) realizaram um estudo de avaliação de desempenho para verificar se o Node.js é uma opção viável para a construção de uma aplicação web moderna. Concluíram que ele demonstra a maior utilização da CPU e supera o PHP por uma amplitude de 2,5 vezes mais rápido em operações de I/O. Além dessas vantagens, a programação orientada a serviços/eventos oferece mais controle sobre a alternância entre as atividades da aplicação. Assim, neste projeto, o Node.js faz o papel de gerenciador dos dados adquiridos e o tratamento dos mesmos, tanto para filtrar como para selecionar adequadamente o que o usuário deseja. Para melhorar o desempenho, foi associado o framework Express, que fornece uma camada de recursos para aplicações sem prejudicar as funcionalidades do Node.js. O Soket.IO foi usado por tornar possível aplicações em tempo real em todos os navegadores e dispositivos móveis, desfocando as diferenças entre os diferentes mecanismos de transporte. No sistema, ele realiza a conexão entre o servidor e o cliente.

O protocolo de comunicação utilizado para comunicar o servidor e os dispositivos é o MQTT. Ele foi escolhido por ser um protocolo de sistema de mensagens assíncrono, que consegue trabalhar com várias requisições ao mesmo tempo. A comunicação síncrona não é adequada às aplicações de IoT, pois, neste tipo de comunicação, uma solicitação só é processada após a finalização da anterior, assim, quando várias requisições são feitas ao mesmo tempo, a fila de tarefas fica grande e demorada (Yuan, 2017). O MQTT foi inventado pensando no modelo Publish/Subscribe, com o propósito de ser leve e capaz de ser utilizado até mesmo por dispositivos restritos e que estejam em redes lentas, com alta latência ou pouco confiáveis (Al-Fuqaha et al., 2015). Neste modelo, quando um elemento da rede deseja receber uma determinada informação, ele a subscreve, fazendo uma requisição para um outro elemento da rede capaz de gerir as publicações e subscrições. Na rede MQTT, este elemento é conhecido como broker, o intermediário no processo de comunicação. Elementos que desejam publicar informações o fazem também através do broker, enviando-lhe as informações que possuem. A identificação das mensagens no MQTT se dá através de tópicos. Elementos da rede podem enviar diversos tópicos para o broker e subscritores podem escolher os tópicos que desejam subscrever. O servidor escolhido para este trabalho, responsável por receber as mensagens do dispositivo, é o Mosquitto, broker de código aberto que implementa as versões 3.1 e 3.1.1 do MQTT. O valor dos sensores é publicado como um dado útil. Os valores fazem parte da mensagem, sendo o formato algo dependente da aplicação, uma vez que o MQTT não impõe restrições sobre isso. Como já mencionado, neste sistema, as mensagens são codificadas no formato JSON e um subscritor pode assinar estas mensagens especificando exatamente o tópico que deseja. No entanto, é mais interessante receber mensagens agrupadas.

A implementação do banco de dados MongoDB foi feita para armazenar dados de todos os dispositivos e ações que passam pelo broker, devido ao seu alto tráfego de dados. O sistema é fundamentado para comunicação em tempo real e a escolha desse banco se deveu ao fato dele ser um banco não relacional rápido e capaz de lidar com o problema da escalabilidade. O MongoDB é orientado a documentos, diferente dos bancos de dados tradicionais que seguem modelo relacional e lidam com registros. Esse banco é estruturado com tabelas independentes e cada uma guarda informações tais como o nome do dispositivo, sua localização, o histórico da atuação, o estado momentâneo, se representa um sinal digital ou analógico etc. Os dados são armazenados em documentos JSON e o formato de consulta também é baseado em JSON.

\subsection{Front-end}

Front-end corresponde à interface de interação com o usuário, ou seja, corresponde ao lado do cliente. 
Todo o sistema foi construído baseado no padrão de arquitetura de software Model-View-Controller (MVC). O model consiste nos dados da aplicação, regras de negócio, lógica e funções. A view corresponde à saída dos dados, podendo ser um valor numérico, uma tabela, um gráfico ou até um diagrama. O controller converte os dados de entrada em comandos para serem enviados ao model ou a view.

A interface foi feita com HyperText Markup Language (HTML) em conjunto com JavaScript e alguns frameworks. O JavaScript foi escolhido porque é uma linguagem interpretada de programação HTML e da web que permite inserir efeitos para que o site fique mais dinâmico e promova uma maior interação com o usuário. Ele também é responsável pela interação com o servidor encarregado da execução dos comandos e sensoriamento. Foi utilizada a biblioteca jQuery para simplificar a programação. O framework Angular.js também foi usado porque facilita a inserção de códigos, funcionando como uma extensão do HTML. Traz o padrão MVC ao cliente, fornecendo a base para aplicativos complexos, ricos e ao mesmo tempo menores, mais rápidos e mais leves. Foram usados ainda dois frameworks: o Bootstrap e o Materialize. O Bootstrap tem código aberto e tem a vantagem de adaptar os projetos web de celulares para tablets e para desktops através de queries. Com função semelhante, o Materialize acelera o desenvolvimento e proporciona beleza à interface.

O front-end do sistema desenvolvido possui as seguintes telas: cliente, usuário, categoria, hardware e dispositivos. Todas essas opções podem ser criadas, atualizadas e deletadas. Na página dos dispositivos, além da possibilidade de criar, editar ou deletar, é possível visualizar por grupos de categoria, localização ou área.

\section{EXPERIMENTOS E RESULTADOS}

\subsection{Software}

O sistema desenvolvido recebeu o nome de "Iotix" e buscou uma front-end simples e intuitiva. Duas telas são mostradas a seguir.

A Figura 2 exibe a tela de controle e monitoramento de dispositivos. Foram realizadas medições da temperatura, umidade, detecção de fumaça e presença e o controle de ventiladores e lâmpadas de cômodos ou abajures. Os dispositivos que aparecem nessa tela são específicos para o cliente de acordo com o cadastro, ou seja, o cliente "A" não terá acesso aos dispositivos associados somente ao cliente "B". É importante destacar ainda que tal controle e monitoramento pode ser realizado por este front-end ou por outro de um sistema que esteja integrado.

A Figura 3 mostra a tela de cadastro de usuários. Nela são requisitados os dados pessoais, tais como nome, e-mail, endereço e a qual cliente está associado. Com esta última informação, identifica-se as funcionalidades que estão disponíveis para cada usuário. As demais telas (clientes, hardwares e categoria) apresentam layout semelhante.

Para testar a aplicabilidade do sistema, foram realizados testes funcionais em todos os campos de formulários. Para o controle e monitoramento dos hardwares conectados ao sistema, foram testados a comunicação, o desempenho e

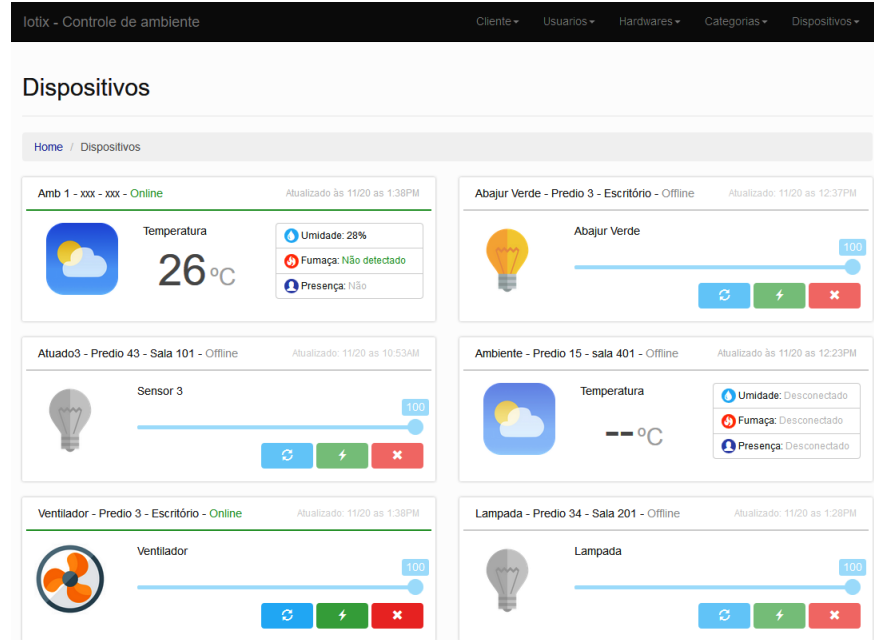

Figura 2. Tela de controle e monitoramento de dispositivos.

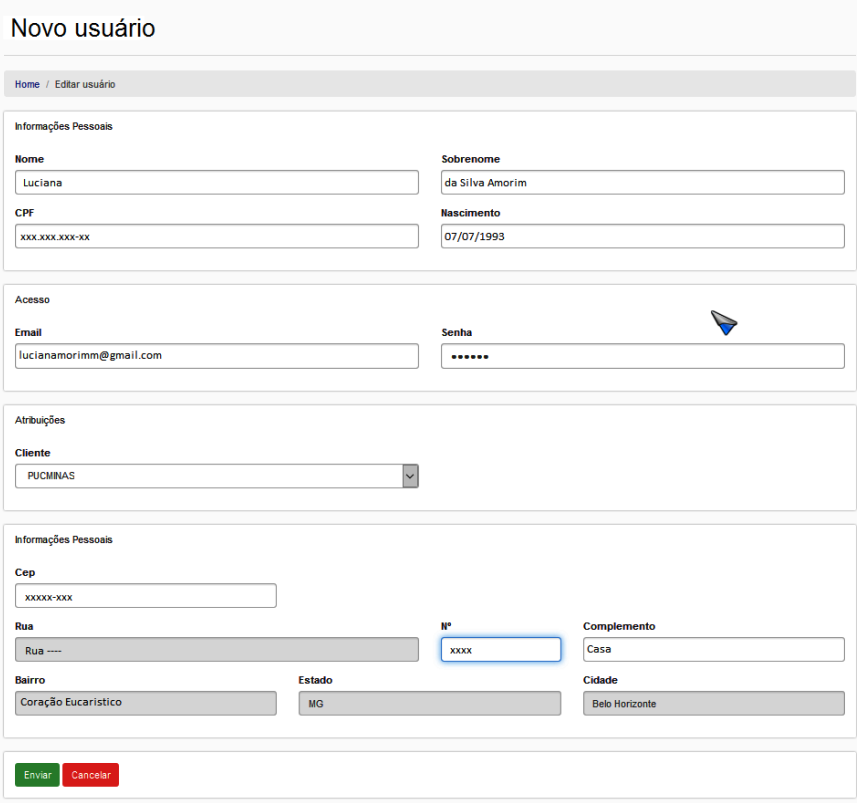

Figura 3. Tela de cadastro de usuários.

a escalabilidade. Através do console do servidor é possível verificar todas as informações referente às requisições, sendo elas HTTP, por websocket e também requisições geradas através do brocker MQTT. Para a comunicação, os dispositivos foram conectados e foram realizados testes de unidade e integração: controle on/off das lâmpadas e ventiladores, medição de temperatura, umidade, presença e detecção de fumaça. Foi possível constatar que a comunicação e os dispositivos estão funcionando como esperado. Para o desempenho, os mesmos testes foram aplicados, porém realizando o monitoramento dos tempos de resposta. Uma vez que o sistema guardou a informação no banco de dados, significa que ele completou a ação de comunicação com o hardware. O teste foi realizado com 10 computadores ligados simultaneamente realizando ações nos dispositivos conectados. O tempo de conclusão das ações, em média, não ultrapassou 20 milissegundos, tanto para as requisições puramente HTTP quanto para as requisições de controle e 
monitoramento do hardware geradas através do broker. No que diz respeito à escalabilidade, foi simulada a presença de 10 sistemas ligados simultaneamente, atentando-se à variação no tempo de resposta do servidor. Pôde-se notar que os tempos de conclusão das ações continuaram praticamente os mesmos, sofrendo influência apenas da qualidade do sinal da internet.

Durantes os testes, todos os dados foram salvos no banco de dados. Mais de 3000 dados foram salvos em menos de 1 minuto. Na realização dos testes, o MongoDB não demonstrou nenhum tipo de anomalia na conexão e o Node.js processou corretamente todas as informações. Esses resultados indicam um sistema estável, com confiabilidade para a plataforma Node.js e para o banco MongoDB. O MQTT garantiu qualidade ao realizar a comunicação em tempo real entre a plataforma e os dispositivos.

Para testar a segurança, foi simulada a ação de um usuário malicioso tentando fazer requisições via MQTT e via HTTP com tokens "errados", gerados aleatoriamente através de um algoritmo. Neste teste, o sistema negou o acesso. Foi testado ainda com um token existente para "usuários comuns". Nesse caso, o usuário tenta utilizar seu token para alterar códigos e realizar tarefas que são bloqueadas para ele. Em todas as tentativas o sistema não permitiu a invasão. Por fim, foi testado o caso das restrições de atividades que antes eram permitidas e agora são bloqueadas para alguns usuários. Nesse caso, o usuário tem um token autenticado, mas agora o sistema deve reconhecê-lo como inválido. O sistema também mostrou segurança nesse teste e impediu a invasão.

Os testes para uso de aplicações externas foram realizados através do software Postman, utilizado para fazer requisições em aplicações. Foram feitas requisições HTTP diretamente ao servidor e este retornou em formato JSON os dados esperados.

Para comprovar a funcionalidade de integração com outros sistemas, foi criado o "CS Universe". Ele é um sistema desenvolvido de forma totalmente independente e, integrado ao Iotix, realizou o controle e monitoramento de algumas funções dos dispositivos. Sua interface foi feita com o framework Polymer. No horário estabelecido pelo usuário, entre as 22:00 h e 6:00 h, por exemplo, se for detectada a presença de movimento no ambiente, será disparado um alarme. Se detectar a presença de fumaça, será mandado um aviso de alerta ao usuário. Pode determinar ainda um horário para acender ou apagar as luzes de forma automática. Em síntese, é um sistema que promove segurança e economia de energia. A front-end do CS é mostrada na Figura 4.

\subsection{Hardware}

Como proposto, os dispositivos foram construídos de maneira independente e podem ser controlados de maneira remota. Possuem tamanho reduzido e são de fácil instalação, proporcionando máxima comodidade e praticidade.

Para realizar a comunicação e controle dos dispositivos, foi necessária a presença do módulo ESP8266. Para favorecer financeiramente, tanto para os desenvolvedores quanto para os clientes, as funções foram agrupadas, de modo que um dispositivo possui mais de uma utilidade. No
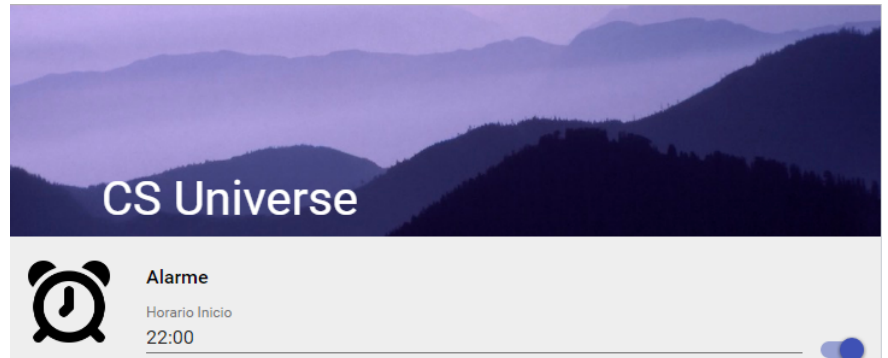

22:00 Horário Termino

06:00

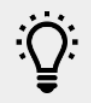

Luz Automática

Horario de Ligar 19:00

Horário de Desligar

06:00

(1)

Controle de Gás

Alerta de presença de gás

Figura 4. Front-end do sistema integrado CS.

mesmo circuito estão presentes o sensor MQ-2 (gás), o sensor DHT11 (umidade e temperatura) e o sensor PIR DYP-ME003 (presença e movimento). O outro dispositivo conta com o relé SRD-05VDC-SL-C para atuar qualquer dispositivo conectado à energia, incluindo o controle de lâmpadas e ventiladores.

$\mathrm{Na}$ Figura 5, é apresentado o dispositivo final com os sensores. Ele está diretamente associado à tela de controle e monitoramento apresentada anteriormente. Os sensores informam as medições na tela e, através dela, podem ser enviados comandos aos atuadores.

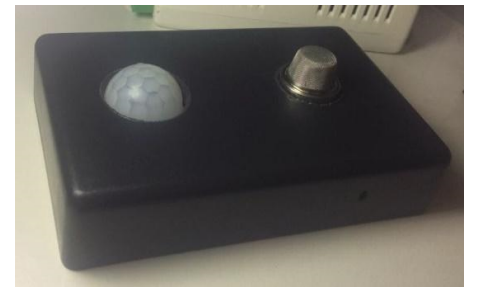

Figura 5. Dispositivo com sensores.

A Figura 6 mostra a visão externa do dispositivo atuador. Para realizar ações sobre ele, basta ligá-lo na rede elétrica do ambiente a ser monitorado e controlado.

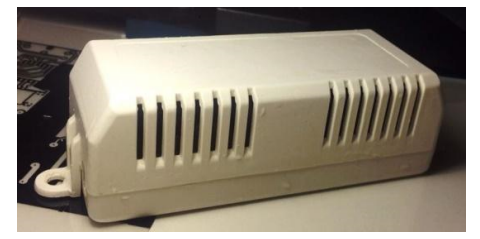

Figura 6. Dispositivo atuador.

\section{CONCLUSÕES E TRABALHOS FUTUROS}

O sistema para controle e monitoramento de ambientes utilizando o conceito de Internet das Coisas foi desenvolvido com o intuito de proporcionar ao usuário praticidade, conforto e segurança. 
A plataforma de desenvolvimento Node.js, o banco de dados MongoDB e o protocolo de comunicação MQTT desempenharam a função proposta como esperado: um sistema nas nuvens que permite a comunicação e o controle dos dispositivos de maneira remota. Suportaram a comunicação em tempo real e a escalabilidade foi atendida. Pôdese notar que todas as requisições tiveram tempos de resposta satisfatórios porque, com a arquitetura distribuída, uma parte do processamento é realizada no lado do servidor e outra parte no lado do cliente, não sobrecarregando o sistema. Com o uso de tokens, o sistema se mostrou seguro tanto para ataques externos quanto para ataques internos.

Futuramente serão incorporados ao sistema dispositivos que realizem o controle da quantidade e da qualidade da energia e sistemas de gerenciamento de informações, como estoque e controle de lixo, por exemplo. O frontend também terá funções implementadas de acordo com a necessidade do cliente.

Para uma proposta de continuidade, é sugerido o desenvolvimento de um sistema que garanta maior nível de machine-to-machine, ou seja, independência dos dispositivos e coisas quanto à ação humana. A arquitetura implantada deve ser baseada em comportamento, não em automação.

\section{REFERÊNCIAS}

Al-Fuqaha, A., Guizani, M., Mohammadi, M., Aledhari, M., e Ayyash, M. (2015). Internet of things: A survey on enabling technologies, protocols, and applications. IEEE Communications Surveys \& Tutorials, 17(4), 2347-2376.

Bigheti, J.A., Caldieri, M.R., e Godoy, E.P. (2016). Automação e controle de processo na nuvem: Proposta e estudo de caso. In XXI Congresso Brasileiro de Automatica.

Brush, A.J., Lee, B., Mahajan, R., Agarwal, S., Saroiu, S., e Dixon, C. (2011). Home automation in the wild: challenges and opportunities. In proceedings of the SIGCHI Conference on Human Factors in Computing Systems, 2115-2124. ACM.

Chaniotis, I.K., Kyriakou, K.I.D., e Tselikas, N.D. (2015). Is node. js a viable option for building modern web applications? a performance evaluation study. Computing, 97(10), 1023-1044.

Chaqfeh, M.A. e Mohamed, N. (2012). Challenges in middleware solutions for the internet of things. In Collaboration Technologies and Systems (CTS), 2012 International Conference on, 21-26. IEEE.

Chess, B. e West, J. (2007). Secure programming with static analysis. Pearson Education.

Coetzee, L. e Eksteen, J. (2011). The internet of thingspromise for the future? an introduction. In IST-Africa Conference Proceedings, 2011, 1-9. IEEE.

Ejaz, W., Naeem, M., Shahid, A., Anpalagan, A., e Jo, M. (2017). Efficient energy management for the internet of things in smart cities. IEEE Communications Magazine, $55(1), 84-91$.

Ferreira, I.V. e Godoy, E.P. (2016). Integracao de internet das coisas e zigbee no contexto de eficiencia energetica e automacao predial. In XXI Congresso Brasileiro de Automatica.
Jain, D., Krishna, P., e Saritha, V. (2012). A study on internet of things based applications. In arXiv preprint arXiv:1206.3891.

Jain, S. e Kajal, A. (2015). Effective analysis of risks and vulnerabilities in internet of things. In International Journal of Computing and Corporate Research, volume 5 .

Karlof, C. e Wagner, D. (2003). Secure routing in wireless sensor networks: Attacks and countermeasures. Ad hoc networks, 1(2-3), 293-315.

Lin, J., Yu, W., Zhang, N., Yang, X., Zhang, H., e Zhao, W. (2017). A survey on internet of things: Architecture, enabling technologies, security and privacy, and applications. IEEE Internet of Things Journal, 4(5), 11251142 .

Lins, V. e Moura, W. (2010). Domótica: automação residencial. In Revista Cientifica Tecnologus, volume 5.

Monteiro, P., Tome, P., e Albuquerque, D. (2015). Domotics control system architecture. In Information Systems and Technologies (CISTI), 2015 10th Iberian Conference on, 1-6. IEEE.

Nath, R.K., Bajpai, R., e Thapliyal, H. (2018). Iot based indoor location detection system for smart home environment. In Consumer Electronics (ICCE), 2018 IEEE International Conference on, 1-3. IEEE.

Rocha Filho, G.P., Mano, L.Y., Valejo, A.D.B., Villas, L.A., e Ueyama, J. (2018). A low-cost smart home automation to enhance decision-making based on fog computing and computational intelligence. IEEE Latin America Transactions, 16(1), 186-191.

Sakurai, L.H., Pisching, M.A., Pessoa, M.A.O., Junqueira, F., e Miyagi, P.E. (2016). Aplicação da internet das coisas em sistemas produtivos com foco na indústria 4.0. In XXI Congresso Brasileiro de Automatica.

Sgarbi, J.A. e Tonidandel, F. (2006). Domótica inteligente: Automação residencial baseada em comportamento. In Workshop de Teses e Dissertacoes em Inteligencia Artificial, Ribeirao Preto, Sao Paulo.

Siano, P., Graditi, G., Atrigna, M., e Piccolo, A. (2013). Designing and testing decision support and energy management systems for smart homes. Journal of Ambient Intelligence and Humanized Computing, 4(6), 651-661.

Teixeira, F.A., Pereira, F., Vieira, G., Marcondes, P., Wong, H.C., Nogueira, J.M.S., e Oliveira, L.B. (2014). Siot-defendendo a internet das coisas contra exploits. In Simpósio Brasileiro de Redes de Computadores e Sistemas Distribuídos (SBRC).

Treter, M.E., Junior, L.P., Franchi, C.M., e Michels, L. (2014). Desenvolvimento de um sistema de automação residencial de baixo custo com acesso remoto via web. In XX Congresso Brasileiro de Automatica.

Yuan, M. (2017). Conhecendo o mqtt: Por que o mqtt é um dos melhores protocolos de rede para a internet das coisas? In IBM. https://www.ibm.com/developerworks/br/library/iotmqtt-why-good-for-iot/index.html. 